\title{
New exponential stability conditions for linear delayed systems of differential equations
}

\author{
Leonid Berezansky ${ }^{1}$, Josef Diblík ${ }^{\otimes 2}$, \\ Zdeněk Svoboda ${ }^{2}$ and Zdeněk Šmarda ${ }^{2}$ \\ ${ }^{1}$ Ben-Gurion University of the Negev, Beer-Sheva 84105, Israel \\ ${ }^{2}$ Brno University of Technology, Technická 10, 61600 Brno, Czech Republic
}

Appeared 11 August 2016

Communicated by Tibor Krisztin

\begin{abstract}
New explicit results on exponential stability, improving recently published results by the authors, are derived for linear delayed systems

$$
\dot{x}_{i}(t)=-\sum_{j=1}^{m} \sum_{k=1}^{r_{i j}} a_{i j}^{k}(t) x_{j}\left(h_{i j}^{k}(t)\right), \quad i=1, \ldots, m
$$

where $t \geq 0, m$ and $r_{i j}, i, j=1, \ldots, m$ are natural numbers, $a_{i j}^{k}:[0, \infty) \rightarrow \mathbb{R}$ are measurable coefficients, and $h_{i j}^{k}:[0, \infty) \rightarrow \mathbb{R}$ are measurable delays. The progress was achieved by using a new technique making it possible to replace the constant 1 by the constant $1+1 /$ e on the right-hand sides of crucial inequalities ensuring exponential stability.
\end{abstract}

Keywords: exponential stability, linear delayed differential system, estimate of fundamental function, Bohl-Perron theorem.

2010 Mathematics Subject Classification: 34K20.

\section{Introduction}

The objective of the present investigation is to derive easily verifiable explicit exponential stability conditions for the following non-autonomous linear delay differential system

$$
\dot{x}_{i}(t)=-\sum_{j=1}^{m} \sum_{k=1}^{r_{i j}} a_{i j}^{k}(t) x_{j}\left(h_{i j}^{k}(t)\right), \quad i=1, \ldots, m
$$

where $t \geq 0, m$ is a natural number, $r_{i j}, i, j=1, \ldots, m$ are natural numbers, the coefficients $a_{i j}^{k}:[0, \infty) \rightarrow \mathbb{R}$ and delays $h_{i j}^{k}:[0, \infty) \rightarrow \mathbb{R}$ are measurable functions.

The equation

$$
\dot{x}(t)=-\sum_{k=1}^{r} a_{k}(t) x\left(h_{k}(t)\right)
$$

\footnotetext{
${ }^{\bowtie}$ Corresponding author. Email: diblik.j@fce.vutbr.cz
} 
which is a special scalar case of (1.1), has been studied, e.g., in [6,12,14,15,20,25]. A review on stability results to equation (1.2) can be found in [7]. Below, we cite some selected results from the above papers or give extracts of them.

From [20, Theorem 1.2], we get the following corollary.

Theorem 1.1. Let there be constants $a_{0}, A_{k}$ and $\tau_{k}, k=1,2, \ldots, r$ such that

$$
0 \leq a_{k}(t) \leq A_{k}, \quad \sum_{k=1}^{r} a_{k}(t) \geq a_{0}>0, \quad 0 \leq t-h_{k}(t) \leq \tau_{k}, \quad t \geq 0 .
$$

If, moreover,

$$
\sum_{k=1}^{r} A_{k} \tau_{k} \leq 1
$$

then the equation (1.2) is uniformly asymptotically stable and the constant 1 on the right-hand side of (1.3) is the best one possible.

A corollary deduced from [20, Theorem 1.1] follows.

Theorem 1.2. Let there be constants $A_{k}$ and $\tau_{k}, k=1,2, \ldots, r$ such that

$$
a_{k}(t) \equiv A_{k}>0, \quad 0 \leq t-h_{k}(t) \leq \tau_{k}, \quad t \geq 0 .
$$

If, moreover,

$$
\sum_{k=1}^{r} A_{k} \tau_{k}<\frac{3}{2}
$$

then the equation (1.2) is uniformly asymptotically stable and the constant $3 / 2$ on the right-hand side of (1.4) is the best one possible.

From [25, Corollary 2.4] we get the following theorem.

Theorem 1.3. Let $a_{k}(t)$ and $h_{k}(t), k=1, \ldots, r, t \geq 0$ be continuous functions and

$$
a_{k}(t) \geq 0, \quad \int_{0}^{\infty} \sum_{k=1}^{r} a_{k}(t) d t=\infty, \quad 0<h_{1}(t) \leq h_{2}(t) \leq \cdots \leq h_{r}(t) \leq t .
$$

If, moreover,

$$
\limsup _{t \rightarrow \infty} \sum_{k=1}^{r} \int_{h_{1}(t)}^{t} a_{k}(s) d s<\frac{3}{2}
$$

then the equation (1.2) is asymptotically stable.

The following result reproduces [15, Proposition 4.4].

Theorem 1.4. Let $a_{k}(t) \equiv a_{k}>0, k=1,2, \ldots, r$ and let a constant $\alpha \in[0,1]$ exist such that

$$
\frac{\alpha}{\mathrm{e} \sum_{i=1}^{r} a_{i}} \leq \max _{k}\left(t-h_{k}(t)\right), \quad t \geq t_{0}
$$

and

$$
\sum_{i=1}^{r} a_{i} \limsup _{t \rightarrow \infty}\left(t-h_{i}(t)\right)<1+\frac{\alpha}{\mathrm{e}}
$$

Then, the equation (1.2) is uniformly asymptotically stable. 
Now we give a corollary of [7, Lemma 3.1].

Theorem 1.5. Let $a_{k}(t)$ be Lebesgue measurable essentially bounded functions and let there be constants $a_{0}$ and $\tau_{k}, k=1,2, \ldots, r$ such that

$$
a_{k}(t) \geq 0, \quad \int_{t_{0}}^{\infty} \sum_{k=1}^{r} a_{k}(s) d s=\infty, \quad 0 \leq t-h_{k}(t) \leq \tau_{k}, \quad t \geq t_{0} .
$$

If, moreover,

$$
\limsup _{t \rightarrow \infty} \sum_{k=1}^{r} \frac{a_{k}(t)}{\sum_{i=1}^{r} a_{i}(t)} \int_{h_{k}(t)}^{t} \sum_{i=1}^{r} a_{i}(s) d s<1+\frac{1}{\mathrm{e}},
$$

then the equation (1.2) is uniformly exponentially stable.

Except for the paper [15], the above mentioned papers consider stability problems for scalar equations only. In [15], linear systems with constant matrices are treated. Unfortunately, there are no results on the stability of general systems of the form (1.1), which can be reduced to Theorems 1.1-1.5 in the scalar case. To illustrate this claim, consider several known results.

In [24], the authors consider the non-autonomous system

$$
\dot{x}_{i}(t)=-\sum_{j=1}^{m} a_{i j}(t) x_{j}\left(h_{i j}(t)\right), \quad i=1, \ldots, m
$$

where $t \in\left[t_{0}, \infty\right), t_{0} \in \mathbb{R}, a_{i j}(t), h_{i j}(t)$ are continuous functions, $h_{i j}(t) \leq t, h_{i j}(t)$ are monotone increasing and such that $\lim _{t \rightarrow \infty} h_{i j}(t)=\infty, i, j=1, \ldots, m$.

Theorem 1.6 ([24, Theorem 2.2]). Assume that, for $t \geq t_{0}$, there exist non-negative numbers $b_{i j}$, $i, j=1, \ldots, m, i \neq j$ such that $\left|a_{i j}(t)\right| \leq b_{i j} a_{i i}(t), i, j=1, \ldots, m, i \neq j, a_{i i}(t) \geq 0$ and

$$
\int^{\infty} a_{i i}(s) d s=\infty, \quad d_{i}=\limsup _{t \rightarrow \infty} \int_{h_{i i}(t)}^{t} a_{i i}(s) d s<3 / 2, \quad i=1, \ldots m .
$$

Let $\tilde{B}=\left(\tilde{b}_{i j}\right)_{i, j=1}^{m}$ be an $m \times m$ matrix with entries $\tilde{b}_{i i}=1, i=1, \ldots, m$ and, for $i \neq j, i, j=1, \ldots, m$,

$$
\tilde{b}_{i j}=\left\{\begin{array}{l}
-\left(\frac{2+d_{i}^{2}}{2-d_{i}^{2}}\right) b_{i j}, \quad \text { if } d_{i}<1, \\
-\left(\frac{1+2 d_{i}}{3-2 d_{i}}\right) b_{i j}, \quad \text { if } \quad d_{i} \geq 1 .
\end{array}\right.
$$

If $\tilde{B}$ is a nonsingular M-matrix, then system (1.6) is asymptotically stable.

This theorem can be viewed as a certain generalization of Theorems 1.2 and 1.3 to systems but only for the case of "one delay" $\left(r_{i j}=1, i, j=1, \ldots, m\right)$.

Paper [13] gives a generalization of Theorem 1.4 to linear systems with constant coefficients and delays.

In our recent paper [8], we considered general system (1.1) deriving the following result.

Theorem 1.7 ([8, Theorem 4]). Let there be constants $a_{0}$ and $\tau$ such that, for $t \geq t_{0}$,

$$
a_{i}^{*}(t):=\sum_{k=1}^{r_{i i}} a_{i i}^{k}(t) \geq a_{0}>0, \quad 0 \leq t-h_{i j}^{k}(t) \leq \tau, \quad i=1, \ldots, m
$$


and

$$
\max _{i=1, \ldots, m} \operatorname{ess} \sup _{t \geq t_{0}} \frac{1}{a_{i}^{*}(t)}\left[\sum_{k=1}^{r_{i i}}\left|a_{i i}^{k}(t)\right| \int_{\max \left\{0, h_{i i}^{k}(t)\right\}}^{t} \sum_{j=1}^{m} \sum_{l=1}^{r_{i j}}\left|a_{i j}^{l}(s)\right| d s+\sum_{\substack{j=1 \\ j \neq i}}^{m} \sum_{\substack{r_{i j} \\ r_{j}}}\left|a_{i j}^{k}(t)\right|\right]<1 .
$$

Then, the system (1.1) is uniformly exponentially stable.

Requiring that all assumptions of Theorem 1.5 and Theorem 1.7 are valid simultaneously, condition (1.8) in Theorem 1.7 turns, in the case of equation (1.2) where $a_{k}(t) \geq 0$, into

$$
\underset{t \geq t_{0}}{\operatorname{ess} \sup _{0}} \frac{1}{\sum_{k=1}^{r} a_{k}(t)} \sum_{k=1}^{r} a_{k}(t) \int_{\max \left\{0, h_{k}(t)\right\}}^{t} \sum_{l=1}^{r} a_{l}(s) d s<1
$$

and, for $t_{0}$ sufficiently large, coincides with the left-hand side of inequality (1.5).

Nevertheless, Theorem 1.7 is not an extension of Theorem 1.5 to system (1.1) since the right-hand side in the inequality (1.8) is equal to 1 instead of $1+1$ /e on the right-hand side of inequality (1.5) in Theorem 1.5.

The aim of the paper is to improve all the results of [8] and replace the constant 1 by the constant $1+1$ /e not only on the right-hand side of inequality (1.8), but in all explicit stability conditions derived in [8]. The only limitation in this paper in comparison with paper [8] is the condition

$$
a_{i i}^{k}(t) \geq 0, \quad i=1, \ldots, m, \quad k=1, \ldots, r_{i i} .
$$

Since this condition does not necessarily hold for equations considered in [8], all results of this paper and in [8] are independent.

Our approach is based on estimates of the fundamental solution for scalar delay differential equations and on the Bohl-Perron type result. Some ideas and schemes of [8] are utilized as well.

\section{Preliminaries}

Let $t_{0} \geq 0$. We consider an initial problem

$$
x(t)=\varphi(t), \quad t \leq t_{0}
$$

for (1.1) where $\varphi=\left(\varphi_{1}, \ldots, \varphi_{m}\right)^{T}:\left(-\infty, t_{0}\right] \rightarrow \mathbb{R}^{m}$ is a vector-function. Throughout the rest of the paper, we assume (a1)-(a3) where

(a1) $a_{i j}^{k}:[0, \infty) \rightarrow \mathbb{R}, i, j=1, \ldots, m, k=1, \ldots, r_{i j}$ are Lebesgue measurable and essentially bounded functions, $a_{i i}^{k}(t) \geq 0$;

(a2) $h_{i j}^{k}:[0, \infty) \rightarrow \mathbb{R}, i, j=1, \ldots, m, k=1, \ldots, r_{i j}$ are Lebesgue measurable functions, $h_{i j}^{k}(t) \leq$ $t$, and $t-h_{i j}^{k}(t) \leq K, t \geq 0$ where $K$ is a positive constant;

(a3) $\varphi:\left(-\infty, t_{0}\right] \rightarrow \mathbb{R}^{m}$ is a Borel measurable bounded vector-function.

For a vector $x=\left(x_{1}, \ldots, x_{m}\right)^{T} \in \mathbb{R}^{m}$, we define $|x|:=\max _{i=1, \ldots, m}\left|x_{i}\right|$. 
Remark 2.1. The function $\varphi$ in (2.1) is defined on $\left(-\infty, t_{0}\right]$. By (a2), there exists a positive constant $K$ such that $t-h_{i j}^{k}(t) \leq K, i, j=1, \ldots, m, k=1, \ldots, r_{i j}$. Thus, the domain of the definition of the initial function $\varphi$ in (2.1) in the following consideration can be, in principle, restricted to the finite interval $\left[t_{0}-K, t_{0}\right]$. In the following computations, it is often necessary to estimate differences $t-\max \left\{t_{0}, h_{i i}^{k}(t)\right\}$ (or similar) from above. Obviously,

$$
t-\max \left\{t_{0}, h_{i i}^{k}(t)\right\} \leq K .
$$

Definition 2.2. A locally absolutely continuous vector-function $x: \mathbb{R} \rightarrow \mathbb{R}^{m}$ is called a solution of the problem (1.1), (2.1) for $t \geq t_{0}$, if its components $x_{i}(t), i=1, \ldots, m$ satisfy (1.1) for almost all $t \in\left[t_{0}, \infty\right)$ and (2.1) holds for $t \leq t_{0}$.

Definition 2.3. Equation (1.1) is called uniformly exponentially stable if there exist constants $M>0$ and $\mu>0$ such that the solution $x: \mathbb{R} \rightarrow \mathbb{R}^{m}$ of (1.1), (2.1) satisfies

$$
|x(t)| \leq M e^{-\mu\left(t-t_{0}\right)} \sup _{t \leq t_{0}}|\varphi(t)|, \quad t \geq t_{0}
$$

where $M$ and $\mu$ do not depend on $t_{0}$.

A non-homogeneous system

$$
\dot{x}_{i}(t)=-\sum_{j=1}^{m} \sum_{k=1}^{r_{i j}} a_{i j}^{k}(t) x_{j}\left(h_{i j}^{k}(t)\right)+f_{i}(t), \quad i=1, \ldots, m
$$

where $f_{i}:[0, \infty) \rightarrow \mathbb{R}$ is a Lebesgue measurable locally essentially bounded function together with the initial problem

$$
x(t)=\theta, \quad t \leq t_{0},
$$

where $\theta=(0, \ldots, 0)^{T} \in \mathbb{R}^{m}$, will be used together with homogeneous system (1.1).

In what follows, $\mathbf{L}_{\infty}^{m}\left[t_{0}, \infty\right)$ denotes the space of all essentially bounded real vectorfunctions $y:\left[t_{0}, \infty\right) \rightarrow \mathbb{R}^{m}$ with the essential supremum norm

$$
\|y\|_{\mathbf{L}_{\infty}^{m}}=\underset{t \geq t_{0}}{\operatorname{ess} \sup }|y(t)|
$$

As $\mathbf{C}^{m}\left[t_{0}, \infty\right)$ we denote the space of all continuous $m$-dimensional bounded real vectorfunctions on $\left[t_{0}, \infty\right)$ equipped with the supremum norm.

The proof of our main result uses the Bohl-Perron type result $([1-5,11,16])$.

Theorem 2.4. If the solution of initial problem (2.2), (2.3) belongs to $\mathbf{C}^{m}\left[t_{0}, \infty\right)$ for any $f \in \mathbf{L}_{\infty}^{m}\left[t_{0}, \infty\right)$, $f=\left(f_{1}, \ldots, f_{m}\right)^{T}$, then equation (1.1) is uniformly exponentially stable.

Note that, without loss of generality, we can assume $f(t) \equiv \theta$ on the interval $\left[t_{0}, t_{1}\right]$ for some $t_{1}>t_{0}$ in Lemma 2.4.

Consider the scalar homogeneous initial problem

$$
\begin{aligned}
& \dot{x}(t)=-\sum_{k=1}^{r} a_{k}(t) x\left(h_{k}(t)\right), \quad t \geq s \geq t_{0}, \\
& x(t)=0, \quad t<s, \quad x(s)=1,
\end{aligned}
$$

where $a_{k}:[0, \infty) \rightarrow \mathbb{R}, k=1, \ldots, r$ are Lebesgue measurable and essentially bounded functions, $h_{k}:[0, \infty) \rightarrow \mathbb{R}, k=1, \ldots, r$ are Lebesgue measurable functions, $h_{k}(t) \leq t$. 
Definition 2.5. A solution $x=X(t, s)$ of (2.4), (2.5) is called the fundamental function of (1.1).

The associated non-homogeneous equation to (2.4) is

$$
\dot{x}(t)=-\sum_{k=1}^{r} a_{k}(t) x\left(h_{k}(t)\right)+f(t), \quad t \geq t_{0} .
$$

We will need the following representation formula (see, e.g. [1-5]) for solution of (2.6) (with a locally Lebesgue integrable right-hand side $f$ ) satisfying the initial problem

$$
x(t)=0, \quad t \leq t_{0} .
$$

Theorem 2.6. The solution of initial problem (2.6), (2.7) is given by the formula

$$
x(t)=\int_{t_{0}}^{t} X(t, s) f(s) d s .
$$

The following lemma is taken from [12].

Theorem 2.7. Let $a_{k}(t) \geq 0$ and

$$
\int_{\min _{k}\left\{h_{k}(t)\right\}}^{t} \sum_{k=1}^{r} a_{k}(s) d s \leq \frac{1}{\mathrm{e}}
$$

where $t \geq t_{0}, k=1, \ldots, r$. Then, the fundamental function $X(t, s)$ of (2.4) satisfies $X(t, s)>0$ for $t \geq s \geq t_{0}$.

We will finish this section by an auxiliary result from [6]. In its formulation, $X(t, s)$ is the fundamental function of (2.4).

Theorem 2.8. Let $a_{k}(t) \geq 0, X(t, s)>0, t \geq s \geq t_{0}, t-h_{k}(t) \leq K, t \geq t_{0}, k=1, \ldots, r$. Then,

$$
0 \leq \int_{t_{0}}^{t} X(t, s)\left(\sum_{k=1}^{r} a_{k}(s)\right) \xi(s) d s \leq 1, \quad t \geq t_{0},
$$

where $\xi$ is the characteristic function of the interval $\left[t_{0}+K, \infty\right)$.

\section{Main result}

The main result (Theorem 3.1 below) gives sufficient conditions for the uniform exponential stability to system (1.1). We underline that this theorem is a significant improvement to Theorem 1.7 because almost the same expression is estimated by the constant $1+1$ /e on the right-hand side of inequality (3.4) rather than by the constant 1 on the right-hand side of inequality (1.8).

Let $A_{i}, i=1, \ldots, m$ be functions defined as

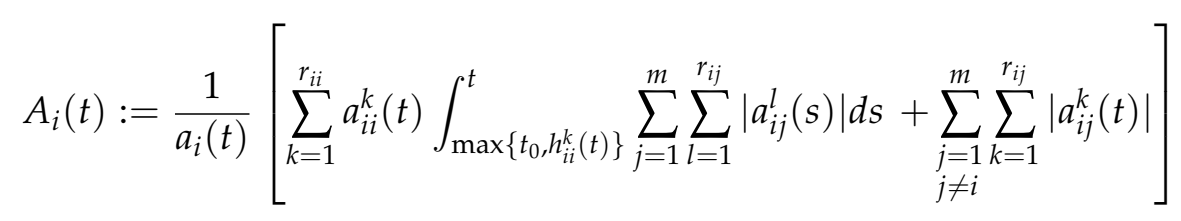

where

$$
a_{i}(t):=\sum_{k=1}^{r_{i i}} a_{i i}^{k}(t)
$$


Theorem 3.1 (Main result). Let

$$
\begin{aligned}
& a_{i}(t) \geq a_{0}>0, \quad i=1, \ldots, m, \quad t \geq t_{0}, \\
& \max _{i=1, \ldots, m} \operatorname{ess} \sup \frac{1}{t^{\prime}(t)} \sum_{\substack{j=1 \\
j \neq i}}^{m} \sum_{k=1}^{r_{i j}}\left|a_{i j}^{k}(t)\right|<1
\end{aligned}
$$

and

$$
\max _{i=1, \ldots, m} \operatorname{ess} \sup _{t \geq t_{0}} A_{i}(t)<1+\frac{1}{\mathrm{e}} .
$$

Then, the system (1.1) is uniformly exponentially stable.

Proof. Define auxiliary functions $H_{i}^{k}:\left[t_{0}, \infty\right) \rightarrow \mathbb{R}, i=1, \ldots, m, k=1, \ldots, r_{i i}$ as follows:

i) If

$$
\int_{h_{i i}^{k}(t)}^{t} \sum_{j=1}^{m} \sum_{l=1}^{r_{i j}}\left|a_{i j}^{l}(s)\right| d s \leq \frac{1}{\mathrm{e}},
$$

then

$$
H_{i}^{k}(t):=h_{i i}^{k}(t)
$$

ii) If

$$
\int_{h_{i i}^{k}(t)}^{t} \sum_{j=1}^{m} \sum_{l=1}^{r_{i j}}\left|a_{i j}^{l}(s)\right| d s>\frac{1}{\mathrm{e}},
$$

then $H_{i}^{k}(t)$ is a unique solution of an implicit equation

$$
\int_{H_{i}^{k}(t)}^{t} \sum_{j=1}^{m} \sum_{l=1}^{r_{i j}}\left|a_{i j}^{l}(s)\right| d s=\frac{1}{\mathrm{e}} .
$$

Consider the problem (2.2), (2.3) assuming that

$$
f_{i}(t) \equiv 0 \quad \text { if } t \in\left[t_{0}, t_{0}+K\right], i=1, \ldots, m .
$$

Condition (3.7) implies that for the solution of the problem (2.2), (2.3) we have $x_{i}(t)=0$, $i=1, \ldots, m$ if $t \in\left[t_{0}, t_{0}+K\right]$.

System (2.2) can be transformed to

$$
\begin{aligned}
\dot{x}_{i}(t)= & -\sum_{k=1}^{r_{i i}} a_{i i}^{k}(t) x_{i}\left(H_{i}^{k}(t)\right)+\sum_{k=1}^{r_{i i}} a_{i i}^{k}(t) \int_{h_{i i}^{k}(t)}^{H_{i}^{k}(t)} \dot{x}_{i}(s) d s \\
& -\sum_{\substack{j=1 \\
j \neq i}}^{m} \sum_{\substack{i j \\
k=1}}^{r_{i j}} a_{i j}^{k}(t) x_{j}\left(h_{i j}^{k}(t)\right)+f_{i}(t), \quad t \geq t_{0}, i=1, \ldots, m .
\end{aligned}
$$

It is easy to see that (due to (2.3)) system (3.8) is equivalent with

$$
\begin{aligned}
\dot{x}_{i}(t)= & -\sum_{k=1}^{r_{i i}} a_{i i}^{k}(t) x_{i}\left(H_{i}^{k}(t)\right)+\sum_{k=1}^{r_{i i}} a_{i i}^{k}(t) \int_{\max \left\{t_{0}, h_{i i}^{k}(t)\right\}}^{H_{i}^{k}(t)} \dot{x}_{i}(s) d s \\
& -\sum_{\substack{j=1 \\
j \neq i}}^{m} \sum_{\substack{i_{i j} \\
j=1}} a_{i j}^{k}(t) x_{j}\left(h_{i j}^{k}(t)\right)+f_{i}(t), \quad t \geq t_{0}, i=1, \ldots, m .
\end{aligned}
$$


Moreover, utilizing (2.2), (3.9), it can be transformed to

$$
\begin{aligned}
\dot{x}_{i}(t)= & -\sum_{k=1}^{r_{i i}} a_{i i}^{k}(t) x_{i}\left(H_{i}^{k}(t)\right) \\
& -\sum_{k=1}^{r_{i i}} a_{i i}^{k}(t) \int_{\max \left\{t_{0}, h_{i i}^{k}(t)\right\}}^{H_{i}^{k}(t)} \sum_{j=1}^{m} \sum_{l=1}^{r_{i j}} a_{i j}^{l}(s) x_{j}\left(h_{i j}^{l}(s)\right) d s \\
& -\sum_{\substack{j=1 \\
j \neq i}}^{m} \sum_{k=1}^{r_{i j}} a_{i j}^{k}(t) x_{j}\left(h_{i j}^{k}(t)\right)+p_{i}(t), \quad t \geq t_{0}, i=1, \ldots, m
\end{aligned}
$$

where

$$
p_{i}(t)=f_{i}(t)+\sum_{k=1}^{r_{i i}} a_{i i}^{k}(t) \int_{\max \left\{t_{0}, h_{i i}^{k}(t)\right\}}^{H_{i}^{k}(t)} f_{i}(s) d s .
$$

By assumption (a2), the definition of $H_{i}^{k}$ (note that $h_{i i}^{k}(t) \leq H_{i}^{k}(t) \leq t$ ), and (3.7) we get

$$
p_{i}(t) \equiv 0 \quad \text { if } t \leq t_{0}+K
$$

Let $X_{i}(t, s), i=1, \ldots, m$ be the fundamental function (see Definition 2.5) of the scalar initialvalue problem

$$
\begin{aligned}
& \dot{x}_{i}(t)=-\sum_{k=1}^{r_{i i}} a_{i i}^{k}(t) x_{i}\left(H_{i}^{k}(t)\right), \quad t \geq t_{0} \\
& x_{i}(t)=0, \quad t \leq t_{0} .
\end{aligned}
$$

By virtue of (a1), the definition of $H_{i}^{k}(t), i=1, \ldots, m$ and Lemma 2.7, we have $X_{i}(t, s)>0$, $t \geq s \geq t_{0}, i=1, \ldots, m$. Using formula (2.8) in Lemma 2.6, from (3.10), we get

$$
\begin{aligned}
x_{i}(t)=-\int_{t_{0}}^{t} X_{i}(t, s) & {\left[\sum_{k=1}^{r_{i i}} a_{i i}^{k}(s) \int_{\max \left\{t_{0}, h_{i i}^{k}(s)\right\}}^{H_{i}^{k}(s)} \sum_{j=1}^{m} \sum_{l=1}^{r_{i j}} a_{i j}^{l}(\tau) x_{j}\left(h_{i j}^{l}(\tau)\right) d \tau\right.} \\
& \left.+\sum_{\substack{j=1 \\
j \neq i}}^{m} \sum_{k=1}^{r_{i j}} a_{i j}^{k}(s) x_{j}\left(h_{i j}^{k}(s)\right)\right] d s+g_{i}(t), \quad t \geq t_{0}, i=1, \ldots, m
\end{aligned}
$$

where

$$
g_{i}(t)=\int_{t_{0}}^{t} X_{i}(t, s) p_{i}(s) d s
$$

and

$$
p_{i}(t)=g_{i}(t) \equiv 0 \quad \text { if } t \leq t_{0}+K
$$

Next, we explain why $g_{i}, i=1, \ldots, m$ are essentially bounded functions. By (a1), properties 
of $f_{i}$ and $H_{i}^{k}, i=1, \ldots, m$, definition (1.7), Remark 2.1, and Lemma 2.8, we deduce

$$
\begin{aligned}
& \text { ess sup }\left|g_{i}(t)\right| \\
& t \geq t_{0} \\
& =\underset{t \geq t_{0}}{\operatorname{ess} \sup }\left|\int_{t_{0}}^{t} X_{i}(t, s) p_{i}(s) d s\right| \\
& =\underset{t \geq t_{0}+K}{\operatorname{ess} \sup }\left|\int_{t_{0}}^{t} X_{i}(t, s) p_{i}(s) d s\right| \\
& \leq \underset{t \geq t_{0}+K}{\operatorname{ess} \sup _{t_{0}}} \int_{i}^{t} X_{i}(t, s) a_{i}(s) \frac{\left|p_{i}(s)\right|}{a_{i}(s)} d s \leq \operatorname{ess~sup}_{t \geq t_{0}+K} \frac{\left|p_{i}(t)\right|}{a_{i}(t)} \\
& \leq \frac{1}{a_{0}} \operatorname{ess~sup}_{t \geq t_{0}+K}\left|p_{i}(t)\right| \\
& \leq \frac{1}{a_{0}}\left(\operatorname{esssup}_{t \geq t_{0}+K}\left|f_{i}(t)\right|+\underset{t \geq t_{0}+K}{\operatorname{ess} \sup _{k}} \sum_{k=1}^{r_{i i}} a_{i i}^{k}(t) \operatorname{ess~sup}_{t \geq t_{0}+K}\left|f_{i}(t)\right| \cdot \underset{t \geq t_{0}+K}{\operatorname{ess} \sup _{i}}\left(H_{i}^{k}(t)-\max \left\{t_{0}, h_{i i}^{k}(t)\right\}\right)\right) \\
& <\infty \text {. }
\end{aligned}
$$

System (3.11) can be written in an operator form

$$
x_{i}(t)=\left(G_{i} x\right)(t)+g_{i}(t), \quad t \geq t_{0}, i=1, \ldots, m
$$

where

$$
\begin{aligned}
\left(G_{i} x\right)(t)=-\int_{t_{0}}^{t} X_{i}(t, s) & {\left[\sum_{k=1}^{r_{i i}} a_{i i}^{k}(s) \int_{\max \left\{t_{0}, h_{i i}^{k}(s)\right\}}^{H_{i}^{k}(s)} \sum_{j=1}^{m} \sum_{l=1}^{r_{i j}} a_{i j}^{l}(\tau) x_{j}\left(h_{i j}^{l}(\tau)\right) d \tau\right.} \\
& \left.+\sum_{\substack{j=1 \\
j \neq i}}^{m} \sum_{k=1}^{r_{i j}} a_{i j}^{k}(s) x_{j}\left(h_{i j}^{k}(s)\right)\right] d s, \quad t \geq t_{0}, i=1, \ldots, m
\end{aligned}
$$

or as

$$
x=G x+g
$$

where

$$
G: \mathbf{L}_{\infty}^{m} \rightarrow \mathbf{L}_{\infty \prime}^{m} \quad(G x)(t)=\left(\left(G_{1} x\right)(t), \ldots,\left(G_{m} x\right)(t)\right)^{T}
$$

and $g(t)=\left(g_{1}(t), \ldots, g_{m}(t)\right)^{T}$. Estimate the norm $\|G\|_{\mathbf{L}_{\infty}^{m}}$ of the operator $G$. Since $x_{i}(t) \equiv 0$, if $t \in\left[t_{0}, t_{0}+K\right], i=1, \ldots, m$, then

$$
\left|\left(G_{i} x\right)(t)\right| \leq \int_{t_{0}+H}^{t} X_{i}(t, s) a_{i}(s) \mathcal{A}_{i}(s) d s \cdot\|x\|_{\mathbf{L}_{\infty^{\prime}}} \quad i=1, \ldots, m
$$

where

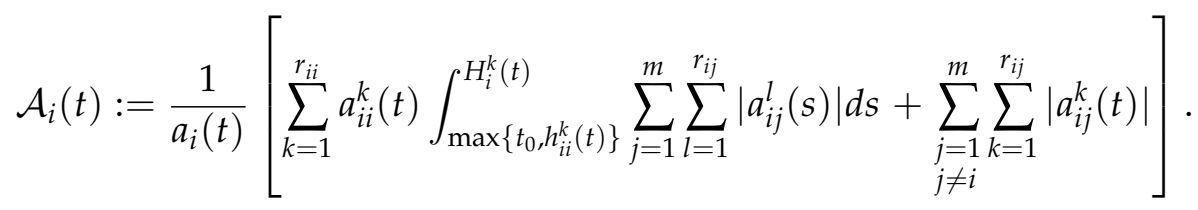

Hence, by Lemma 2.8,

$$
\|G\|_{L_{\infty}^{m}} \leq \max _{i=1, \ldots, m} \operatorname{ess} \sup \mathcal{A}_{i}(t)
$$


If (3.5) holds, then $H_{i}^{k}(t)=h_{i i}^{k}(t), i=1, \ldots, m, k=1, \ldots, r_{i i}$ and, consequently,

$$
\mathcal{A}_{i}(t) \leq \frac{1}{a_{i}(t)}\left[\sum_{\substack{j=1 \\ j \neq i}}^{m} \sum_{\substack{r_{i j} \\ j=1}}\left|a_{i j}^{k}(t)\right|\right]
$$

By (3.3) we get

$$
\max _{i=1, \ldots, m} \operatorname{ess} \sup \mathcal{A}_{i}(t) \leq \max _{i=1, \ldots, m} \operatorname{ess} \sup \frac{1}{t \geq t_{0}} \frac{1}{a_{i}(t)}\left[\sum_{\substack{j=1 \\ j \neq i}}^{m} \sum_{k=1}^{r_{i j}}\left|a_{i j}^{k}(t)\right|\right]<1 .
$$

If (3.6) is valid, then

$$
\int_{H_{i}^{k}(t)}^{t} \sum_{j=1}^{m} \sum_{l=1}^{r_{i j}}\left|a_{i j}^{l}(s)\right| d s=\frac{1}{\mathrm{e}} .
$$

Hence

$$
\begin{aligned}
& \frac{1}{a_{i}(t)} \sum_{k=1}^{r_{i i}} a_{i i}^{k}(t) \int_{\max \left\{t_{0}, h_{i i}^{k}(t)\right\}}^{H_{i}^{k}(t)} \sum_{j=1}^{m} \sum_{l=1}^{r_{i j}}\left|a_{i j}^{l}(s)\right| d s \\
& =\frac{1}{a_{i}(t)} \sum_{k=1}^{r_{i i}} a_{i i}^{k}(t)\left[\int_{\max \left\{t_{0}, h_{i i}^{k}(t)\right\}}^{t} \sum_{j=1}^{m} \sum_{l=1}^{r_{i j}}\left|a_{i j}^{l}(s)\right| d s-\int_{H_{i}^{k}(t)}^{t} \sum_{j=1}^{m} \sum_{l=1}^{r_{i j}}\left|a_{i j}^{l}(s)\right| d s\right] \\
& =\frac{1}{a_{i}(t)} \sum_{k=1}^{r_{i i}} a_{i i}^{k}(t)\left[\int_{\max \left\{t_{0}, h_{i i}^{k}(t)\right\}}^{t} \sum_{j=1}^{m} \sum_{l=1}^{r_{i j}}\left|a_{i j}^{l}(s)\right| d s-\frac{1}{\mathrm{e}}\right] \\
& =\frac{1}{a_{i}(t)} \sum_{k=1}^{r_{i i}} a_{i i}^{k}(t) \int_{\max \left\{t_{0}, h_{i i}^{k}(t)\right\}}^{t} \sum_{j=1}^{m} \sum_{l=1}^{r_{i j}}\left|a_{i j}^{l}(s)\right| d s-\frac{1}{\mathrm{e}} .
\end{aligned}
$$

In this case, using (3.15) and (3.4), we get

$$
\max _{i=1, \ldots, m} \underset{t \geq t_{0}}{\operatorname{ess} \sup } \mathcal{A}_{i}(t) \leq \max _{i=1, \ldots, m} \underset{\operatorname{ess} \sup }{t \geq t_{0}}\left(A_{i}(t)-\frac{1}{\mathrm{e}}\right)<1 .
$$

Finally, from (3.13), (3.14) and (3.16), we deduce $\|G\|_{\mathbf{L}_{\infty}^{m}}<1$. Therefore, the operator equation (3.12) has a unique solution $x \in \mathbf{L}_{\infty}^{m}$ This solution solves the system (2.2) and belongs to the space $\mathbf{C}^{m}\left[t_{0}, \infty\right)$. By Lemma 2.4 , system (1.1) is uniformly exponentially stable.

\section{Corollaries to the main result}

The purpose of this part is to consider some special cases of the system (1.1) and from Theorem 3.1, deduce simple corollaries on uniform exponential stability. In the proofs, we verify the assumptions of Theorem 3.1 for the case considered. It is often obvious and we omit the unnecessary details.

Corollary 4.1. Assume that

$$
a_{i i}(t) \geq a_{0}>0, \quad i=1, \ldots, m, \quad t \geq t_{0},
$$




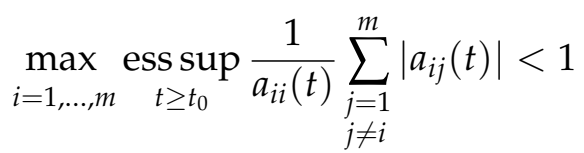

and

$$
\max _{i=1, \ldots, m} \operatorname{ess} \sup _{t \geq t_{0}}\left[\int_{\max \left\{t_{0}, h_{i i}(t)\right\}}^{t} \sum_{j=1}^{m}\left|a_{i j}(s)\right| d s+\frac{1}{a_{i i}(t)} \sum_{\substack{j=1 \\ j \neq i}}^{m}\left|a_{i j}(t)\right|\right]<1+\frac{1}{\mathrm{e}} .
$$

Then, the system

$$
\left.\dot{x}_{i}(t)=-\sum_{j=1}^{m} a_{i j}(t) x_{i}\left(h_{i j}(t)\right)\right), \quad i=1, \ldots, m
$$

is uniformly exponentially stable.

Proof. Let $r_{i j}=1, a_{i j}^{k}(t)=a_{i j}(t), h_{i j}^{k}(t)=h_{i j}(t), a_{i}(t)=a_{i i}(t), i, j=1, \ldots, m$. Then, the system (1.1) reduces to (4.4) and we can apply Theorem 3.1 since assumptions (3.2), (3.3) and (3.4) are, in the particular case, reduced to assumptions (4.1), (4.2) and (4.3).

Corollary 4.2. Assume that, for $t \geq t_{0}$, we have $a_{i i}^{k}(t) \geq 0$,

$$
\sum_{k=1}^{r_{i i}} a_{i i}^{k}(t) \geq \alpha_{i}>0, \quad\left|a_{i j}^{k}(t)\right| \leq a_{i j}^{k}, \quad t-h_{i j}^{k}(t) \leq \tau_{i j}^{k}
$$

where $i, j=1, \ldots, m, k=1, \ldots, r_{i j}, \alpha_{i}, a_{i j}^{k}, \tau_{i j}^{k}$ are constants,

$$
\max _{i=1, \ldots, m} \frac{1}{\alpha_{i}} \sum_{\substack{j=1 \\ j \neq i}}^{m} \sum_{k=1}^{r_{i j}} a_{i j}^{k}<1,
$$

and

$$
\max _{i=1, \ldots, m} \frac{1}{\alpha_{i}}\left[\left(\sum_{k=1}^{r_{i i}} a_{i i}^{k} \tau_{i i}^{k}\right)\left(\sum_{j=1}^{m} \sum_{l=1}^{r_{i j}} a_{i j}^{l}\right)+\sum_{\substack{j=1 \\ j \neq i}}^{m} \sum_{k=1}^{r_{i j}} a_{i j}^{k}\right]<1+\frac{1}{\mathrm{e}} .
$$

Then, the system (1.1) is uniformly exponentially stable.

Proof. We have for $t \geq t_{0}$

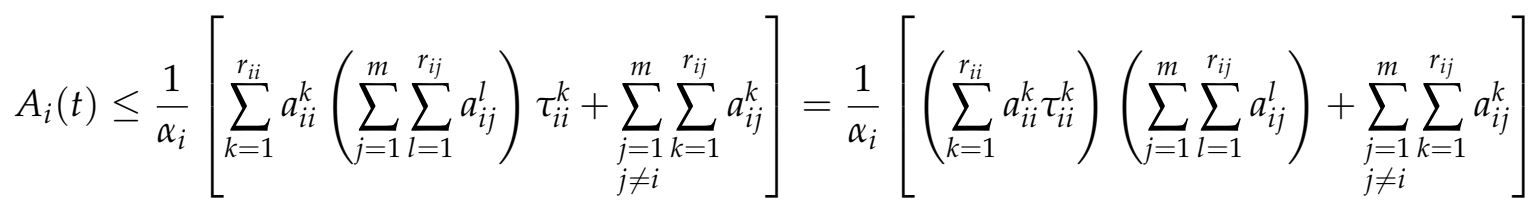

and (4.6) implies (3.4).

Corollary 4.3. Assume that $a_{i i}(t) \geq \alpha_{i}>0,\left|a_{i j}(t)\right| \leq a_{i j}, t-h_{i j}(t) \leq \tau_{i j}$ for $i, j=1, \ldots, m$ and $t \geq t_{0}$ where $\alpha_{i}, a_{i j}$, and $\tau_{i j}$ are constants and

$$
\max _{i=1, \ldots, m} \frac{1}{\alpha_{i}} \sum_{\substack{j=1 \\ j \neq i}}^{m} a_{i j}<1, \quad \max _{i=1, \ldots, m}\left[\tau_{i i} \sum_{j=1}^{m} a_{i j}+\frac{1}{\alpha_{i}} \sum_{\substack{j=1 \\ j \neq i}}^{m} a_{i j}\right]<1+\frac{1}{\mathrm{e}} .
$$

Then, the system (4.4) is uniformly exponentially stable. 
Proof. This result follows from Corollary 4.1.

Now we give stability conditions for the following linear autonomous system with constant delays

$$
\dot{x}_{i}(t)=-\sum_{j=1}^{m} \sum_{k=1}^{r_{i j}} a_{i j}^{k} x_{j}\left(t-\tau_{i j}^{k}\right), \quad i=1, \ldots, m .
$$

Corollary 4.4. Assume that $a_{i i}^{k} \geq 0$, conditions (4.5) and (4.6) hold where

$$
\alpha_{i}:=\sum_{k=1}^{r_{i i}} a_{i i}^{k}>0, \quad i=1, \ldots, m .
$$

Then, the autonomous system (4.8) is uniformly exponentially stable.

Proof. This follows directly from Corollary 4.2.

Consider the linear autonomous system with constant delays

$$
\dot{x}_{i}(t)=-\sum_{j=1}^{m} a_{i j} x_{j}\left(t-\tau_{i j}\right), \quad i=1, \ldots, m .
$$

Corollary 4.5. Assume that $a_{i i}>0$ and inequalities (4.7) hold where $\alpha_{i}=a_{i i}, i=1, \ldots, m$. Then, the autonomous system (4.9) is uniformly exponentially stable.

Proof. This follows directly from Corollary 4.3.

Corollary 4.6. Assume that $m=1, a_{k}(t) \geq 0, k=1, \ldots, r$ and, for $t \geq t_{0}$, at least one of the following conditions hold $\left(a_{0}, a_{i}\right.$ and $\tau_{i}, i=1, \ldots, r$ are constants):

1) $\sum_{k=1}^{r} a_{k}(t) \geq a_{0}>0$,

$$
\underset{t \geq t_{0}}{\operatorname{ess} \sup _{0}} \frac{1}{\sum_{k=1}^{r} a_{k}(t)}\left[\sum_{k=1}^{r} a_{k}(t) \int_{\max \left\{t_{0}, h_{k}(t)\right\}}^{t} \sum_{l=1}^{r} a_{l}(s) d s\right]<1+\frac{1}{\mathrm{e}} .
$$

2) $a_{i}(t) \equiv a_{i}, \sum_{i=1}^{r} a_{i}>0, t-h_{i}(t) \leq \tau_{i}, i=1, \ldots, r$, and

$$
\sum_{i=1}^{r} a_{i} \tau_{i}<1+\frac{1}{\mathrm{e}}
$$

Then, the scalar equation (1.2) is uniformly exponentially stable.

Proof. Let condition 1) be true. Then, inequality (3.4) turns into inequality (4.10) for $m=1$. Let condition 2) be true. Since $a_{i}(t) \equiv a_{i}$, inequality (4.10) is transformed to

$$
\underset{t \geq t_{0}}{\operatorname{ess} \sup _{k=1}} \sum_{k=1}^{r} a_{k}\left(t-\max \left\{t_{0}, h_{k}(t)\right\}\right)<1+\frac{1}{\mathrm{e}} .
$$

Since

$$
\underset{t \geq t_{0}}{\operatorname{ess} \sup } \sum_{k=1}^{r} a_{k}\left(t-\max \left\{t_{0}, h_{k}(t)\right\}\right) \leq \underset{t \geq t_{0}}{\operatorname{ess} \sup } \sum_{k=1}^{r} a_{k} \tau_{k}
$$

inequality (4.11) implies (4.10). 
Now we consider two particular cases of system (1.1),

$$
\dot{X}(t)=-B(t) X(h(t))
$$

and

$$
\dot{X}(t)=-A(t) X(t)-B(t) X(h(t))
$$

where $A(t)=\left(a_{i j}(t)\right)_{i, j=1}^{m}, B(t)=\left(b_{i j}(t)\right)_{i, j=1}^{m}$ are $m \times m$ matrices with Lebesgue measurable and locally essentially bounded entries

$$
a_{i j}:[0, \infty) \rightarrow \mathbb{R}, \quad b_{i j}:[0, \infty) \rightarrow \mathbb{R}, \quad i, j=1, \ldots, m
$$

and $X(t)=\left(x_{1}(t), \ldots, x_{m}(t)\right)^{T}$. Assume that, for the delay $h:[0, \infty) \rightarrow \mathbb{R}$, the relevant adaptation of condition (a2) holds, i.e., $h$ is Lebesgue measurable, $h(t) \leq t$ and $t-h(t) \leq K, t \in[0, \infty)$ and $\lim \sup _{t \rightarrow \infty}(t-h(t))<\infty$.

The following two Corollaries 4.7 and 4.8 deal with the exponential stability of systems (4.12), (4.13).

Corollary 4.7. Assume that, for $t \geq t_{0}$, at least one of the conditions hold $\left(b_{0}, \tau, \alpha_{i}\right.$ and $b_{i j}^{*}, i, j=$ $1, \ldots, r$ are constants):

a) $b_{i i}(t) \geq b_{0}>0, i=1, \ldots, m$,

$$
\max _{i=1, \ldots, m} \operatorname{ess} \sup _{t \geq t_{0}} \frac{1}{b_{i i}(t)} \sum_{\substack{j=1 \\ j \neq i}}^{m}\left|b_{i j}(t)\right|<1,
$$

and

$$
\max _{i=1, \ldots, m} \operatorname{ess} \sup _{t \geq t_{0}}\left[\int_{\max \left\{t_{0}, h(t)\right\}}^{t} \sum_{j=1}^{m}\left|b_{i j}(s)\right| d s+\frac{1}{b_{i i}(t)} \sum_{\substack{j=1 \\ j \neq i}}^{m}\left|b_{i j}(t)\right|\right]<1+\frac{1}{\mathrm{e}} .
$$

b) $b_{i i}(t) \geq \alpha_{i}>0,\left|b_{i j}(t)\right| \leq b_{i j^{\prime}}^{*} t-h(t) \leq \tau, i, j=1, \ldots, m$,

$$
\max _{i=1, \ldots, m} \frac{1}{\alpha_{i}} \sum_{\substack{j=1 \\ j \neq i}}^{m} b_{i j}^{*}<1, \quad \max _{i=1, \ldots, m}\left[\tau \sum_{j=1}^{m} b_{i j}^{*}+\frac{1}{\alpha_{i}} \sum_{\substack{j=1 \\ j \neq i}}^{m} b_{i j}^{*}\right]<1+\frac{1}{\mathrm{e}} .
$$

Then, the system (4.12) is uniformly exponentially stable.

Proof. System (4.12) can be written in the form

$$
\dot{x}_{i}(t)=-\sum_{j=1}^{m} b_{i j}(t) x_{j}(h(t)), \quad i=1, \ldots, m .
$$

Now, the corollary directly follows from Corollaries 4.1 and 4.3.

Corollary 4.8. Assume that, for $t \geq t_{0}$,

$$
a_{i i}(t) \geq 0, \quad b_{i i}(t) \geq 0, \quad a_{i i}(t)+b_{i i}(t) \geq a_{0}>0, \quad i=1, \ldots, m,
$$


where $a_{0}$ is a constant,

$$
\max _{i=1, \ldots, m} \operatorname{esssup} \frac{1}{t \geq t_{0}} \frac{1}{a_{i i}(t)+b_{i i}(t)} \sum_{\substack{j=1 \\ j \neq i}}^{m}\left(\left|a_{i j}(t)\right|+\left|b_{i j}(t)\right|\right)<1,
$$

and

$$
\begin{aligned}
& \max _{i=1, \ldots, m} \operatorname{esssup}_{t \geq t_{0}} \frac{1}{a_{i i}(t)+b_{i i}(t)}\left[b_{i i}(t) \int_{\max \left\{t_{0}, h(t)\right\}}^{t} \sum_{j=1}^{m}\left(\left|a_{i j}(s)\right|+\left|b_{i j}(s)\right|\right) d s+\sum_{\substack{j=1 \\
j \neq i}}^{m}\left(\left|a_{i j}(t)\right|+\left|b_{i j}(t)\right|\right)\right] \\
& \quad<1+\frac{1}{\mathrm{e}} .
\end{aligned}
$$

Then, the system (4.13) is uniformly exponentially stable.

Proof. We can write system (4.13) as

$$
\left.\dot{x}(t)=-\sum_{j=1}^{m} a_{i j}(t) x_{j}(t)\right)-\sum_{j=1}^{m} b_{i j}(t) x_{j}(h(t)), \quad i=1, \ldots, m
$$

and use Theorem 3.1 for the choice $r_{i i}=2, a_{i j}^{1}(t)=a_{i j}(t), a_{i j}^{2}(t)=b_{i j}(t), h_{i j}^{1}(t)=t, h_{i j}^{2}(t)=h(t)$, $i, j=1, \ldots, m$. Hence, $a_{i}(t)=a_{i i}(t)+b_{i i}(t), i=1, \ldots, m$ and inequality (4.14) coincides with (3.4).

Consider particular cases of systems (4.12), (4.13)

$$
\dot{X}(t)=-B X(t-\tau)
$$

and

$$
\dot{X}(t)=-A X(t)-B X(t-\tau)
$$

where $A=\left(a_{i j}\right)_{i, j=1}^{m}$ and $B=\left(b_{i j}\right)_{i, j=1}^{m}$ are constant matrices, $\tau>0$, and $a_{i i} \geq 0, b_{i i} \geq 0$, $i=1, \ldots, m$.

Corollary 4.9. Assume that $b_{i i}>0, i=1,2, \ldots, m$, and

$$
\max _{i=1, \ldots, m} \frac{1}{b_{i i}} \sum_{\substack{j=1 \\ j \neq i}}^{m}\left|b_{i j}\right|<1, \quad \max _{i=1, \ldots, m}\left[\tau \sum_{j=1}^{m}\left|b_{i j}\right|+\frac{1}{b_{i i}} \sum_{\substack{j=1 \\ j \neq i}}^{m}\left|b_{i j}\right|\right]<1+\frac{1}{\mathrm{e}} .
$$

Then, the system (4.15) is uniformly exponentially stable.

Proof. This follows from Corollary 4.7 (b) where $\alpha_{i}=b_{i i}$.

Corollary 4.10. Assume that $a_{i i} \geq 0, b_{i i} \geq 0, a_{i i}+b_{i i}>0$,

$$
\frac{1}{a_{i i}+b_{i i}} \sum_{\substack{j=1 \\ j \neq i}}^{m}\left(\left|a_{i j}\right|+\left|b_{i j}\right|\right)<1
$$

and

$$
\frac{1}{a_{i i}+b_{i i}}\left[\tau b_{i i} \sum_{j=1}^{m}\left(\left|a_{i j}\right|+\left|b_{i j}\right|\right)+\sum_{\substack{j=1 \\ j \neq i}}^{m}\left(\left|a_{i j}\right|+\left|b_{i j}\right|\right)\right]<1+\frac{1}{\mathrm{e}}
$$

for $i=1, \ldots, m$. Then, the system (4.16) is uniformly exponentially stable. 
Proof. Estimating the left-hand side of inequality (4.14) in the case of system (4.16) and using (4.17), we obtain

$$
\begin{aligned}
& \max _{i=1, \ldots, m} \underset{\operatorname{mit}}{\operatorname{ess} \sup } \frac{1}{a_{i i}(t)+b_{i i}(t)}\left[b_{i i}(t) \int_{\max \left\{t_{0}, h(t)\right\}}^{t} \sum_{j=1}^{m}\left(\left|a_{i j}(s)\right|+\left|b_{i j}(s)\right|\right) d s+\sum_{\substack{j=1 \\
j \neq i}}^{m}\left(\left|a_{i j}(t)\right|+\left|b_{i j}(t)\right|\right)\right] \\
& \leq \max _{i=1, \ldots, m} \frac{1}{a_{i i}+b_{i i}}\left[\tau b_{i i} \sum_{j=1}^{m}\left(\left|a_{i j}\right|+\left|b_{i j}\right|\right)+\sum_{\substack{j=1 \\
j \neq i}}^{m}\left(\left|a_{i j}\right|+\left|b_{i j}\right|\right)\right]<1+\frac{1}{\mathrm{e}} .
\end{aligned}
$$

Therefore, inequality (4.14) holds and Corollary 4.10 is a consequence of Corollary 4.8.

\section{Concluding remarks}

First we will compare the stability results obtained in the paper with some known result. Let system (1.1) be of the form

$$
\begin{aligned}
& \dot{x}_{1}(t)=-a_{11}(t) x_{1}\left(h_{11}(t)\right)-a_{12}(t) x_{2}\left(h_{12}(t)\right), \\
& \dot{x_{2}}(t)=-a_{21}(t) x_{1}\left(h_{21}(t)\right)-a_{22}(t) x_{2}\left(h_{22}(t)\right) .
\end{aligned}
$$

Here, $m=2$ and $r_{i j}=1, i, j=1,2$. Assume that there are constants $\alpha_{i}, A_{i j}, \tau_{i j}, i, j=1,2$ such that $0<\alpha_{i} \leq a_{i i}(t),\left|a_{i j}(t)\right| \leq A_{i j}$ and $t-h_{i j}(t) \leq \tau_{i j} \leq K$ and, for a constant $q \in(0,1)$, $\left|a_{12}(t)\right| \leq q a_{11}$ and $\left|a_{21}(t)\right| \leq q a_{22}, t \in\left[t_{0}, \infty\right)$. Then, (3.2) and (3.3) hold. Inequality (3.4) holds if

$$
\begin{aligned}
& \left(A_{11}+A_{12}\right) \tau_{11}+\frac{A_{12}}{\alpha_{1}}<1+\frac{1}{\mathrm{e}}, \\
& \left(A_{22}+A_{21}\right) \tau_{22}+\frac{A_{21}}{\alpha_{2}}<1+\frac{1}{\mathrm{e}} .
\end{aligned}
$$

By Theorem 3.1, system (5.1) is uniformly exponential stable. The above assumptions are valid, e.g., for the choice

$$
a_{i i}(t) \equiv A_{i i}=\alpha_{i}=0.1, \quad a_{i j}(t) \equiv A_{i j}=0.099, \quad i \neq j, \quad \tau_{i j}=1.89
$$

in (5.1) if $i, j=1,2$.

Apply Theorem 1.6 if $t-h_{i j}(t) \equiv \tau_{i j} \leq K, a_{i i}(t) \equiv A_{i i}=\alpha_{i}>0, a_{i j}(t) \equiv A_{i j}$ if $i \neq j$, $i, j=1,2$ in (5.1). Let $0<a_{12}=b_{12} a_{11}$ and $0<a_{21}=b_{21} a_{22}, t \in\left[t_{0}, \infty\right)$. We get $d_{i}=A_{i i} \tau_{i i}$, $i=1$, 2 . If $d_{i}<1$, then

$$
\begin{aligned}
& \tilde{b}_{12}=-\left(\frac{2+A_{11}^{2} \tau_{11}^{2}}{2-A_{11}^{2} \tau_{11}^{2}}\right) \frac{A_{12}}{A_{11}}, \\
& \tilde{b}_{21}=-\left(\frac{2+A_{22}^{2} \tau_{22}^{2}}{2-A_{22}^{2} \tau_{22}^{2}}\right) \frac{A_{21}}{A_{22}} .
\end{aligned}
$$

Theorem 1.6 implies (recall that a square matrix is a nonsingular $M$-matrix if its inverse is a positive matrix)) the following result. If

$$
A_{i i} \tau_{i i}<1, \quad \tilde{b}_{12} \tilde{b}_{21}<1,
$$

then system (5.1) is asymptotically stable. 
Let (5.3) is set in (5.1). Then,

$$
A_{i i} \tau_{i i}=0.189<1, \quad \tilde{b}_{12} \tilde{b}_{21} \doteq 1.053 \nless 1
$$

and Theorem 1.6 is not applicable.

It is not difficult to derive examples when conditions (5.2) hold, but stability conditions of another known results are not valid.

The stability conditions derived in the paper are written in the form of inequalities with the right-hand sides which are equal the constant $1+1$ /e. As we mentioned in the introduction, the purpose of this paper was to improve all the results of [8] with the extra condition (1.9). The first open problem is to remove this condition in all statements of this paper.

Nevertheless, there is another challenge for a possible continuation of investigations. Analysing some stability results (e.g. [18, Theorem 5.9]) where in the inequalities considered, the constant $3 / 2$ plays a significant role as a non-improvable bound, an open problem arises, if we can expect that our results can be improved by replacing the constant $1+1$ /e by the constant $3 / 2$ in the inequalities used. An alternative problem is to prove or disprove that, for the general case of variable coefficients and delays, the constant $1+1$ /e is the best one possible.

For further results on the stability of linear delay differential systems, we refer, e.g., to the review paper [23] and to [19,21]. Recent results on global asymptotic stability for delay differential systems can be found in $[9,10,17,22]$.

Another research challenge is the following. In this paper and in all known papers on the stability of linear delay differential systems, the conditions sufficient for stability involve only diagonal delays. It will be interesting to obtain stability conditions such that all delays are utilized in the relevant inequalities.

As noted in [8], only few necessary stability conditions are known for systems. One of the interesting problems is the following. To prove or disprove the following conjecture: if system (1.1) is asymptotically stable, then the sum of the diagonal elements is nonnegative, i.e.,

$$
\sum_{i=1}^{m} \sum_{k=1}^{r_{i i}} a_{i i}^{k}(t) \geq 0, \quad t \geq t_{0}
$$

Finally, we recall a problem tacitly mentioned in the introduction - for system (1.1), derive stability results that could be reduced to Theorems 1.1-1.5 in the scalar case.

\section{Acknowledgements}

The second and the third author were supported by the Grant 201/11/0768 of the Czech Grant Agency (Prague). The fourth author was supported by the grant FEKT-S-14-2200 of Faculty of Electrical Engineering and Communication, Brno University of Technology.

\section{References}

[1] N. V. Azbelev, L. Berezansky, P. M. Simonov, A. V. Chistyakov, The stability of linear systems with aftereffect. I, Differential Equations 23(1987), No. 5, 493-500. MR0896353

[2] N. V. Azbelev, L. Berezansky, P. M. Simonov, A. V. Chistyakov, The stability of linear systems with aftereffect. II, Differential Equations 27(1991), No. 4, 383-388. MR1120152 
[3] N. V. Azbelev, L. Berezansky, P. M. Simonov, A. V. Chistyakov, The stability of linear systems with aftereffect. III, Differential Equations 27(1991), No. 10, 1165-1172. MR1157670

[4] N. V. Azbelev, L. Berezansky, P. M. Simonov, A. V. Chistyakov, The stability of linear systems with aftereffect. IV, Differential Equations 29 (1993), no. 2, 153-160. MR1236101

[5] N. V. Azbelev, P. M. Simonov, Stability of differential equations with aftereffect, Stability and Control: Theory, Methods and Applications, Vol. 20, Taylor \& Francis, London, 2003. MR1965019

[6] L. Berezansky, E. Braverman, On exponential stability of linear differential equations with several delays, J. Math. Anal. Appl. 324(2006), 1336-1355. MR2266563; url

[7] L. Berezansky, E. Braverman, New stability conditions for linear differential equations with several delays, Abstr. Appl. Anal. 2011, Art. ID 178568, 19 pp. MR2800075; url

[8] L. Berezansky, J. Diblík, Z. Svoboda, Z. Šmarda, Simple uniform exponential stability conditions for a system of linear delay differential equations, Appl. Math. Comput. 250(2015), 605-614. MR3285565; url

[9] T. FARIA, Asymptotic behaviour for a class of delayed cooperative models with patch structure, Discrete Contin. Dyn. Syst. Ser. B 18(2013), No. 6, 1567-1579. MR3038769; url

[10] T. FARIA, J. J. Oliveira, General criteria for asymptotic and exponential stabilities of neural network models with unbounded delays, Appl. Math. Comput. 217(2011), No. 23, 96469658. MR2811239; url

[11] M. GIL', Stability of vector differential delay equations, Frontiers in Mathematics, Birkhäuser/Springer Basel AG, Basel, 2013. MR3026099

[12] S. A. Gusarenko, A. I. Domoshnitski, Asymptotic and oscillation properties of firstorder linear scalar functional-differential equations, Differential Equations 25(1989), No. 12, 1480-1491. MR1044645

[13] I. GYőRI, F. HARTung, Fundamental solution and asymptotic stability of linear delay differential equations, Dyn. Contin. Discrete Impuls. Syst. Ser. A Math. Anal. 13(2006), No. 2, 261-287. MR2204331

[14] I. GYőRI, F. HARTUNG, Stability in delay perturbed differential and difference equations, in: Topics in functional differential and difference equations (Lisbon, 1999), Fields Inst. Commun., Vol. 29, Amer. Math. Soc., Providence, RI, 2001, 181-194. MR1821781

[15] I. GYőRI, F. Hartung, J. TuRI, Preservation of stability in delay equations under delay perturbations, J. Math. Anal. Appl. 220(1998), 290-312. MR1613964; url

[16] A. Halanay, Differential equations: Stability, oscillations, time lags, Academic Press, New York-London, 1966. MR0216103

[17] L. V. Hien, V. N. Phat, H. Trinh, New generalized Halanay inequalities with applications to stability of nonlinear non-autonomous time-delay systems, Nonlinear Dynamics 82(2015), No. 1-2. MR3405665; url 
[18] V. KolmanovskiI, A. MyshKis, Introduction to the theory and applications of functionaldifferential equations, Mathematics and its Applications, Vol. 463, Kluwer Academic Publishers, Dordrecht, 1999. MR1680144

[19] V. Kolmanovskit, L. Shaikhet, About one application of the general method of Lyapunov functionals construction, Internat. J. Robust Nonlinear Control 13(2003), No. 9, 805-818. MR1998313; url

[20] T. KRISZTIN, On stability properties for one-dimensional functional-differential equations, Funkcial. Ekvac. 34(1991), 241-256. MR1130462

[21] K. Liu, V. Suplin, E. Fridman, Stability of linear systems with general sawtooth delay, IMA J. Math. Control Inform. 27(2010), No. 4, 419-436. MR2762983; url

[22] E. Liz, A. Ruiz-Herrera, Attractivity, multistability, and bifurcation in delayed Hopfield's model with non-monotonic feedback, J. Differential Equations 255(2013), No. 11, 4244-4266. MR3097255; url

[23] J.-P. RichaRd, Time-delay systems: An overview of some recent advances and open problems, Automatica 39(2003), No. 10, 1667-1694. MR2141765; url

[24] J. W.-H. So, X. H. TANG, X. Zou, Global attractivity for non-autonomous linear delay systems, Funkcial. Ekvac. 47(2004), No. 1, 25-40. MR2075286

[25] J. W.-H. So, J.S. Yu, M.-P. CHEN, Asymptotic stability for scalar delay differential equations, Funkcial. Ekvac. 39(1996), No. 1, 1-17. MR1401650 